\title{
Application of Brillouin Optical Correlation Domain Analysis for Crack Identification in Concrete Structure
}

\begin{abstract}
Michio IMAI ${ }^{*}$ and Satoru MIURA
Kajima Technical Research Institute, Tokyo, 1820036, Japan

*Corresponding author: Michio IMAIＥ-mail: michio@kajima.com

Abstract: This paper investigates the application of distributed optical fiber strain sensors to civil engineering structures, because no other tool can satisfactorily detect the location of the unpredictable phenomenon. In fact, the locations of cracks in the concrete structure are unknown $a$ priori; therefore, a fully distributed sensor is necessary to detect them. The Brillouin optical correlation domain analysis (BOCDA), which offers high spatial resolution by using stimulated Brillouin scattering along the whole length of the optical fiber, is used in a wide range of civil engineering applications, and the same has undergone significant development over the last decade. In this paper, it is demonstrated how a BOCDA-based strain sensor can be employed to monitor cracks in concrete. Crack monitoring on the surface of the concrete member provides useful information for evaluating stiffness and durability of the structure, particularly for early detection of tiny cracks, which is essential for preventing crack growth and dispersion. The crack-induced strain distribution was analytically investigated, and it was proved that BOCDA can identify even a small crack before its visual recognition by a beam test. Moreover, periodical crack monitoring was successfully executed on a pedestrian deck for five years.
\end{abstract}

Keywords: Stimulated Brillouin scattering, optical fiber sensor, strain measurement, distributed sensing

Citation: Michio IMAI and Satoru MIURA, "Application of Brillouin Optical Correlation Domain Analysis for Crack Identification in Concrete Structure," Photonic Sensors, DOI: 10.1007/s13320-013-0134-3.

\section{Introduction}

Degradation of the civil infrastructure has been receiving much attention for a decade in Japan, because a large number of concrete structures are more than fifty years old [1]. Unfortunately, aging of structures is outpacing the growth of the maintenance budget. Structural assessment methods of these structures are still dominantly based on labor-intensive and time-consuming measurement, e.g. visual inspection or tapping test. Against this background, structural health monitoring (SHM) can be regarded as one of the best candidates, not only for risk management, but also for life cycle management. In the civil engineering field, the optical fiber sensor has undergone tremendous development because of its unique advantages, such as the light weight, small diameter, electro-magnetic immunity, and long lifetime [2]. In comparison to many other types of optical fiber sensors, the Brillouin-based sensor, which has been recently studied, can provide more valuable information, i.e. a spatially continuous strain distribution profile along an installed optical fiber. Therefore, the Brillouin-based sensor can detect strain changes even in the most unexpected locations.

Received: 21 July 2013 / Revised version: 1 September 2013

(C) The Author(s) 2013. This article is published with open access at Springerlink.com 
As a method of Brillouin-based sensors, the Brillouin optical time domain reflectometer (BOTDR) covers a long measurement range by an injected pulse light wave, while Brillouin optical correlation domain analysis (BOCDA) shows high spatial resolution by changing the frequency of two-encountered continuous light waves [3]. Though their advantages and drawbacks are different, both the sensors are good enough to perform SHM in terms of strain distribution measurement. Having originated from the telecommunications technology, the BOTDR has successfully emerged to be a competent monitor of earthen structures or long structures, e.g. the deformation of a levee [4] or the displacement of a pipeline [5]. On the other hand, BOCDA is developed to detect even a tiny strain change within the range of the limited measurement length, thanks to its high spatial resolution. Figure 1 illustrates a BOCDA-based sensor setup, using an injected laser diode with synchronized pulse modulation [6].

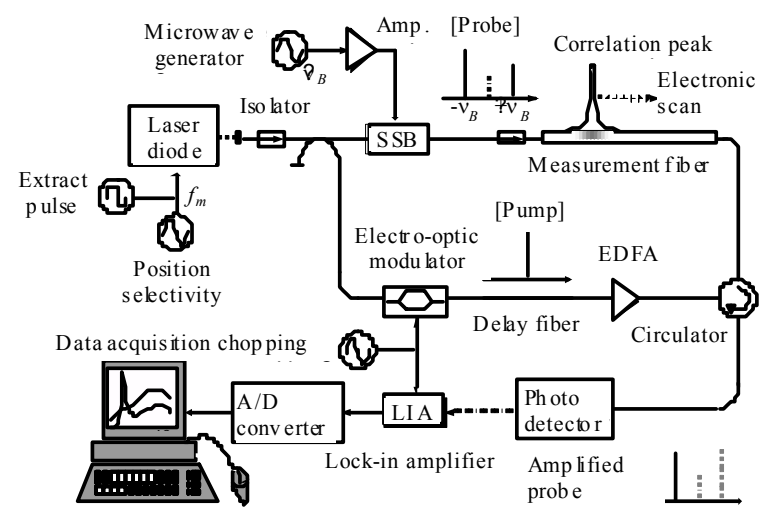

Fig. 1 BOCDA-based optical fiber strain sensor setup.

Unexpected cracking of the concrete is a frequent complaint. The crack can be the result of one or a combination of complex factors: drying shrinkage, thermal contraction, restraint (external or internal) to shortening, subgrade settlement, applied loads, and fatigue. Concrete cracking causes several problems, such as low stiffness, low durability, and poor appearance. It should be noted that degradation factors, which come into play, via crack, affect the concrete member more than the crack itself, by way of accelerating the corrosion of rebar. Therefore, knowing the cause and behavior of crack is important in assessing current and future structural status.

This paper describes the experimental study carried out by using BOCDA to monitor cracks, which were developed in a beam specimen in the laboratory and a bridge deck in the field, by mounting an optical fiber sensor on the concrete surface.

\section{Crack identification methodology}

\subsection{Strain distribution model}

Once a crack occurs on a concrete surface, the infinite strain generates on the mounted sensing optical fiber if it is perfectly fixed on the surface, and the fiber is susceptible to the breakage. However, the mounted fiber would survive, because the applied stress is less than the stress on the concrete surface owing to the buffer provided by the surrounding materials, i.e. the adhesive and the fiber protective jacket. Also, the sensor's limited spatial resolution can possibly affect the measurement sensitivity. In this work, it is assumed that crack identification from strain distributions measured by the optical fiber strain sensor involves the following:

(1) Finite element analysis to understand the buffering material effect, followed by obtaining the applied strain distribution on the sensing segment of the fiber, $S_{\text {fiber }}(z)$.

(2) Estimation of the measured strain distribution, $S_{\text {meas. }}(z)$, assuming the Gaussian distribution of the sensor's sensitivity.

Considering half of the cracked concrete surface, a two-dimensional concrete prism was modeled, which is partially shown in Fig. 2. The displacement of the top end of a sensing optical fiber element corresponds to half of the crack width, whereas the bottom and left parts of the concrete surface were fixed as the boundary condition. Both the optical fiber (with the Young's modulus of $1.0 \times 10^{5} \mathrm{MPa}$ and 
Poisson ratio of 0.2 ) and the concrete (with the Young's modulus of $3.0 \times 10^{4} \mathrm{MPa}$ and Poisson ratio of 0.16) were modeled as quadrilateral plane stress elements. Between the optical fiber and the concrete elements, the buffering elements, which comprised the epoxy adhesive and the fiber protective jacket, were considered together. Considering the material property given in the earlier study [7], the Young's modulus and shear modulus of the buffering element were taken to be $1,000 \mathrm{MPa}$ and $160 \mathrm{MPa}$, respectively.

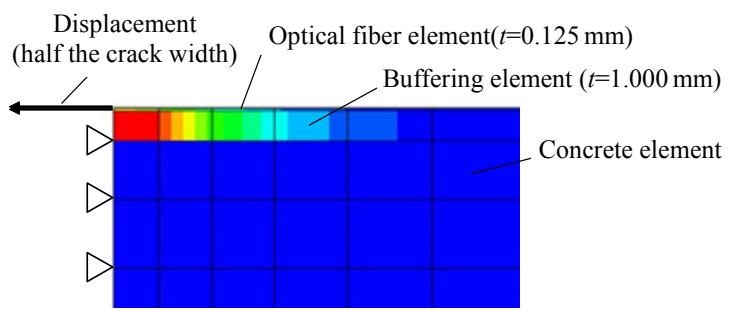

Fig. 2 2D finite element model enlarged partially.

Figure 3 shows the analytical results for increasing the crack width, where the zero position corresponds to the center of the crack, expressing the strain distribution along the sensing fiber, $S_{\text {fiber }}(z)$. As the existing numerical study indicates [8], it can be seen that the strain at the tip of the crack increases with an increase in the width of the crack in the figure. Besides, the area affected by the crack does not change because of the lack of separation or slippage in the buffering materials. Within $40 \mu \mathrm{m}$ of the crack, it is empirically found that such nonlinear phenomena are negligible [9]. Cracks wider than $40 \mu \mathrm{m}$ are visible and are hence beyond the scope of this study.

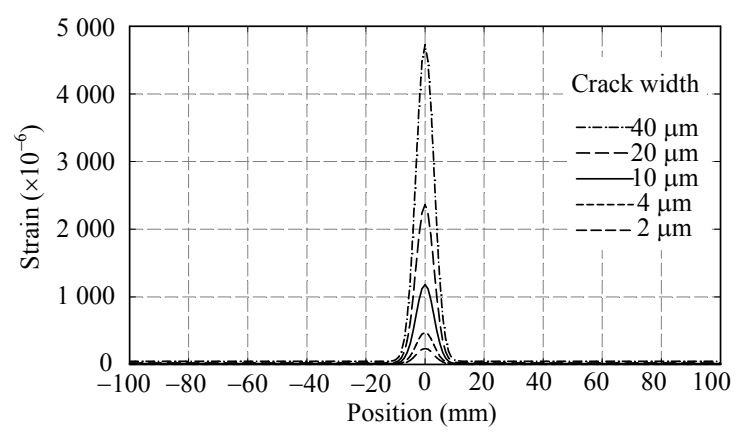

Fig. 3 Analyzed strain distribution along the fiber, $S_{\text {fiber }}(z)$.

The applied strain distribution on the sensing segment was calculated using the above mentioned finite element (FE) analysis. However, some information relating to the strain distribution measured by optical fiber sensors would be lost owing to the limited spatial resolution. To consider such an effect, the following calculation was employed. Although the sensitivity distribution of BOCDA is, in principle, expressed by the accumulation of megahertz sinusoidal modulation of the lightwaves, it was simply considered to be the Gaussian distribution, as having been the practice in many practical applications. The standard deviation from the distribution is equal to $14.5 \mathrm{~mm}$, which corresponds to half of the spatial resolution $\Delta z$ used in the beam test described later.

$S_{\text {meas. }}(z)=\int_{-\infty}^{\infty} S_{\text {fiber }}(l) \cdot \frac{1}{\sqrt{2 \pi}(\Delta z / 2)} \exp \left(-\frac{(l-z)^{2}}{2(\Delta z / 2)^{2}}\right) d l$.

Figure 4 shows the analyzed strain distribution after considering all buffering effects, $S_{\text {meas. }}(z)$. One readily observes that the affected area reaches around $60 \mathrm{~mm}$, despite the small size of the crack. According to the measurement precision of the BOCDA-based sensor, $\pm 100 \times 10^{-6}$ approximately, it is even possible to detect cracks that are more than $10 \mu \mathrm{m}$ as a peak-shaped strain distribution.

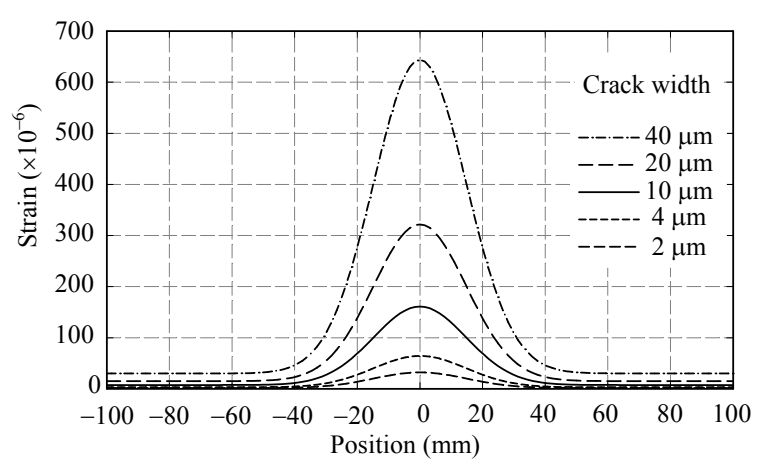

Fig. 4 Estimated strain distribution, $S_{\text {meas. }}(z)$.

\subsection{BOCDA measurement on FRC beam test}

To validate the above mentioned crack identification method, a four-point bending test on a beam was conducted. As depicted in Fig. 5, a T-shaped specimen was fabricated from three steel pre-stressed concrete (PC) wires and fiber reinforced 
concrete (FRC). The specimen was $3560 \mathrm{~mm}$ long, $250 \mathrm{~mm}$ high, and $404 \mathrm{~mm}$ wide. An optical fiber was mounted as the sensing medium on the bottom girder surface by means of a two-component epoxy adhesive (see Fig. 6).

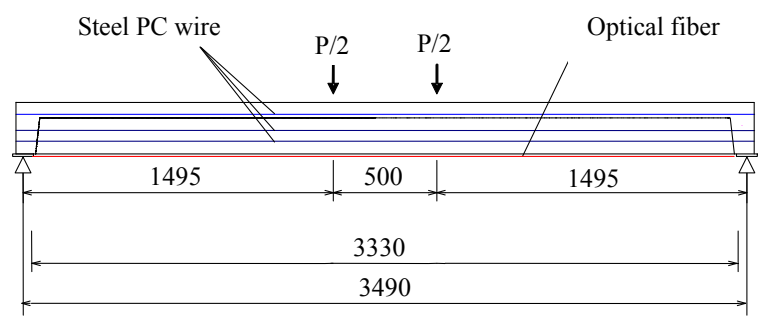

(a) Longitudinal section 404

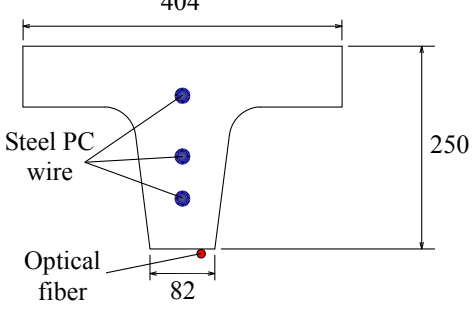

(b) Cross section

Fig. 5 FRC beam specimen (dimensions in $\mathrm{mm}$ ).

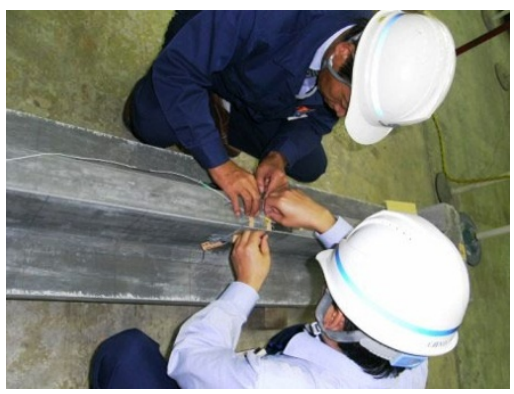

Fig. 6 Optical fiber sensor installation on the specimen.

Figure 7 shows the experimental setup of the four-point bending test on the beam. The distances between the two supports and between the two loading points were $3490 \mathrm{~mm}$ and $500 \mathrm{~mm}$, respectively. The measurement range for the optical fiber sensor was $3330 \mathrm{~mm}$ along the middle of the span. The longitudinal strain was measured statically by the BOCDA-based sensor at 19-mm measurement intervals while maintaining the load for a couple of minutes. In this case, the spatial resolution was $29 \mathrm{~mm}$. Once a crack was detected by the sensor, it was observed under the microscope, primarily because there is no other alternative to investigating an imperceptible crack in the FRC.
Going by the results of previous studies on the FRC, the first crack was expected to appear at a load of $74 \mathrm{kN}$.

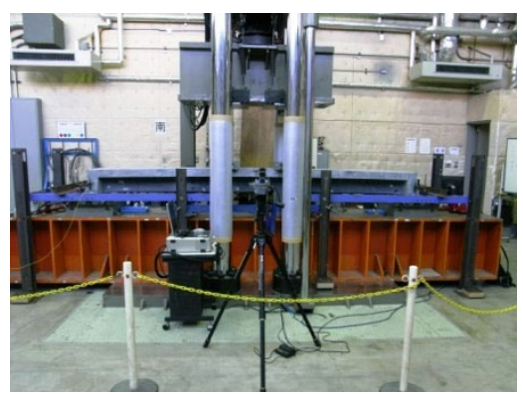

Fig. 7 Four-point bending experiment.

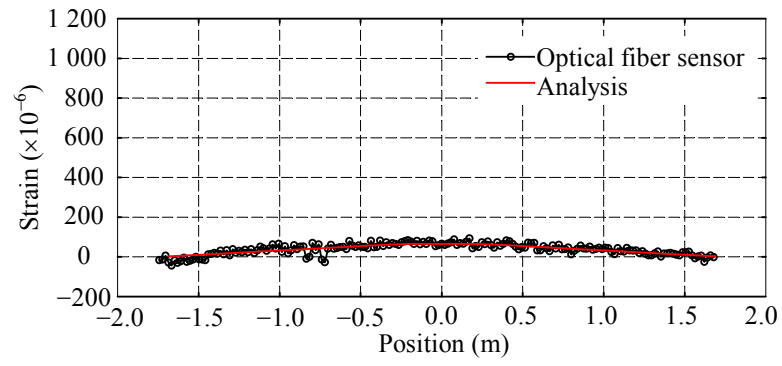

(a) At $5 \mathrm{kN}$

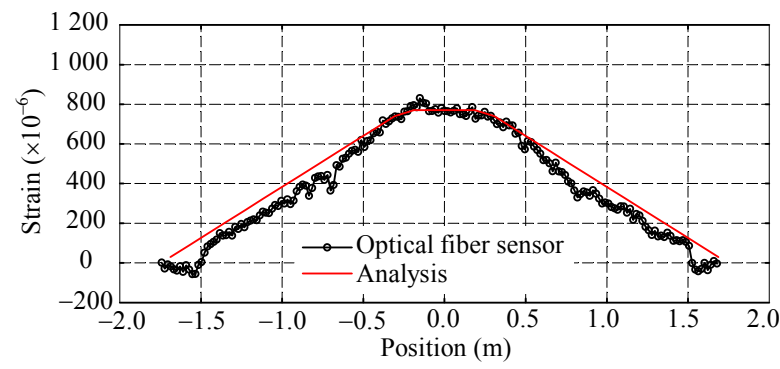

(b) At $60 \mathrm{kN}$

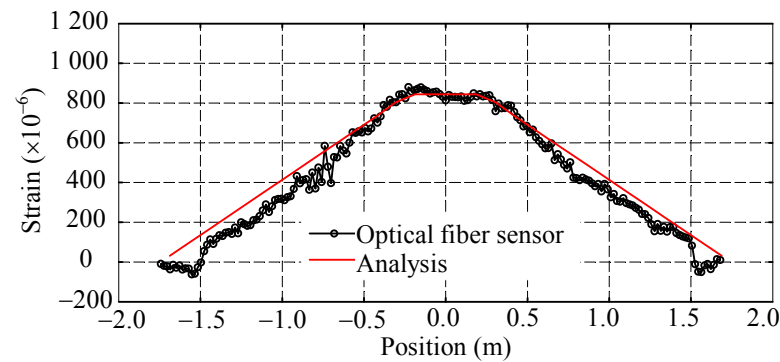

(c) At $65 \mathrm{kN}$

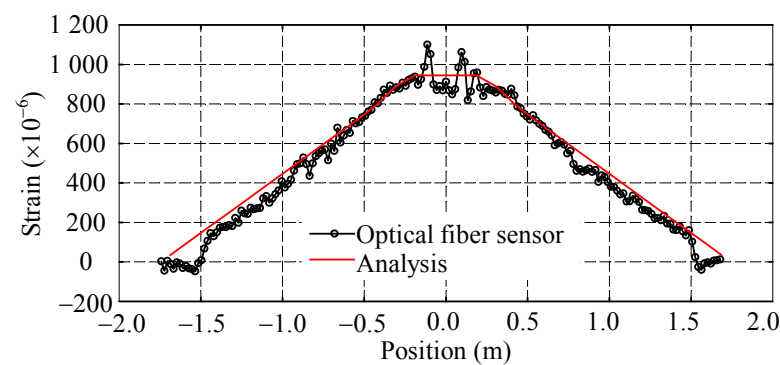

(d) At $70 \mathrm{kN}$

Fig. 8 Strain distribution measured by BOCDA. 
The measured and analyzed results are shown in Figs. 8 (a) -8 (d). The measurements are in good agreement with the analytical results, and the peak-shaped strain distributions appear at $70 \mathrm{kN}$, around the middle of the span. The location of the peak corresponds to the location of the crack observed under the microscope (see Fig. 9); hence, the three strain peaks in Fig. 8 (d) are confirmed to be derived from three discrete cracks on the bottom surface of the FRC specimen.

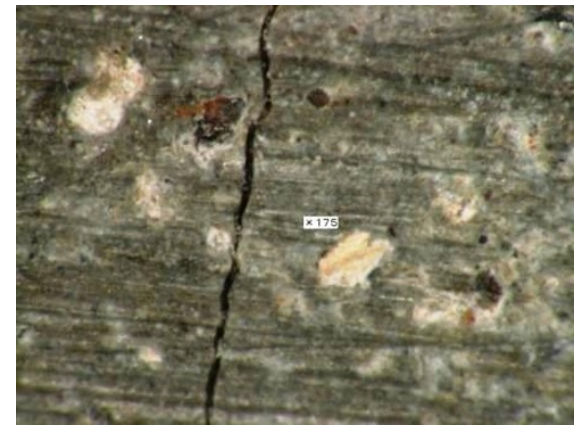

Fig. 9 Microscopic image of tiny crack.

\subsection{Crack identification}

From the time the first crack was detected by the optical fiber sensor, the crack width can be determined using the strain distribution model expressed by (1), because the test was carried out in the same manner as the analysis in terms of installation and measurement settings. Figure 9 shows the enlarged strain distribution around the middle of the span, along with the back-calculated crack profile. At a load of $70 \mathrm{kN}$, appropriate profiles can be observed in all the three cracks, as shown in Fig. 10 (a). Based on the modeled crack profile, the following cracks can be identified: Crack 1: $14 \mu \mathrm{m}$ at $-0.110 \mathrm{~m}$; Crack 2: $15 \mu \mathrm{m}$ at $+0.095 \mathrm{~m}$; Crack 3: $8 \mu \mathrm{m}$ at $+0.180 \mathrm{~m}$. This identification is in reasonable agreement with the microscope observations. At $75 \mathrm{kN}$, however, the difference between the measurement and modeled profile is significant for Crack 2, as shown in Fig. 10 (b). Between $70 \mathrm{kN}$ and $75 \mathrm{kN}$, new cracks were generated adjacent to the existing ones, and because the sensor could not discriminate multiple cracks, the crack width was underestimated. Overall, the model for the crack-induced strain distribution is valid for a single crack within the range of about $60 \mathrm{~mm}$, which is almost twice the spatial resolution.

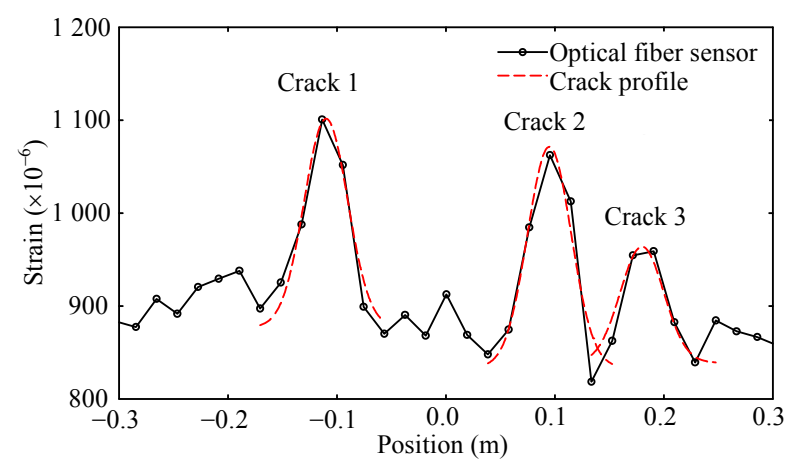

(a) At $70 \mathrm{kN}$

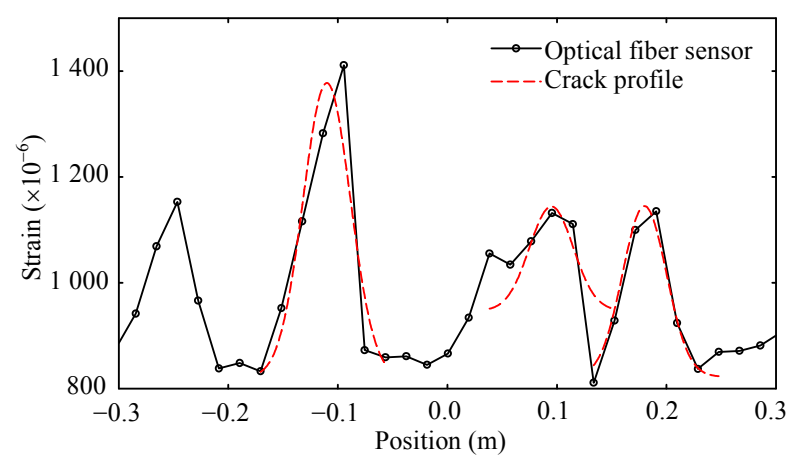

(b) At $75 \mathrm{kN}$

Fig. 10 Crack identification by strain distribution.

At $70 \mathrm{kN}, 75 \mathrm{kN}$, and $80 \mathrm{kN}$, the widths of the original two cracks were identified by the modeled profile, and the results were compared with microscope observations (see Fig. 11). Although it is necessary to consider the effect of multiple cracks to identify a crack individually, the proposed strain

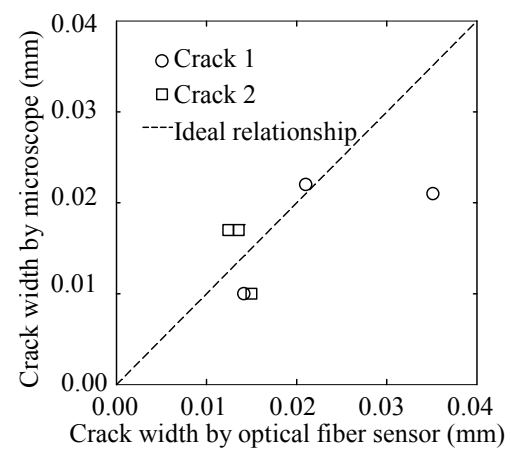

Fig. 11 Comparison between the crack width measured by BOCDA and those by microscope. 
distribution model can be efficiently considered applicable to the single crack.

\subsection{Results and discussion}

The four-point bending test was conducted on a 3.5-m FRC beam specimen. During the loading, the longitudinal strain distribution was measured by a BOCDA-based optical fiber sensor with a spatial resolution of $29 \mathrm{~mm}$. The measurements agree well with those at the analytical bending moments; besides, the discrete cracks in the concrete can be detected from the peak-like shapes of crack-induced strain distribution curves. It was also experimentally confirmed that the crack widths and locations calculated from the strain distribution model are reasonably in accord with the observed values.

\section{Crack-monitoring application}

\subsection{Pedestrian deck overview}

For crack-monitoring applications, the BOCDAbased sensor was applied to a pedestrian deck in the northern part of Japan. As depicted in Fig. 12, the span was $2 m+26 m+2 m$, the deck's width was $4.1 \mathrm{~m}$, and the girder's height was $500 \mathrm{~mm}$. The structural element was composed of the FRC, which was pre-stressed by four external cables. In the summer of 2007, just after the completion of the

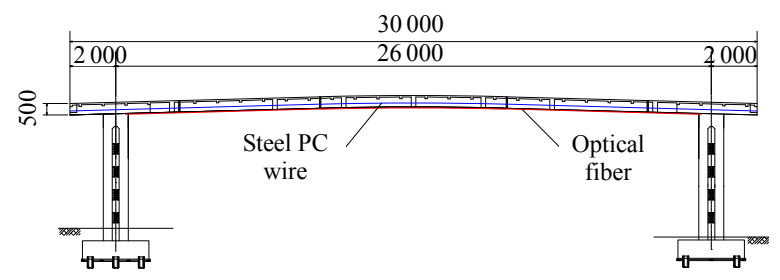

(a) Longitudinal section

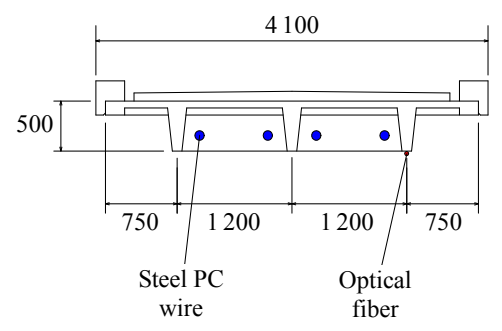

(b) Cross section

Fig. 12 Pedestrian deck (dimensions in $\mathrm{mm}$ ). deck construction, an optical fiber was mounted as the sensing medium, as in the case of the beam-bending test, on the bottom surface of the girder at the mid-span using a two-component epoxy adhesive (see Fig. 13). The measurement region along the girder was approximately $24 \mathrm{~m}$ long.

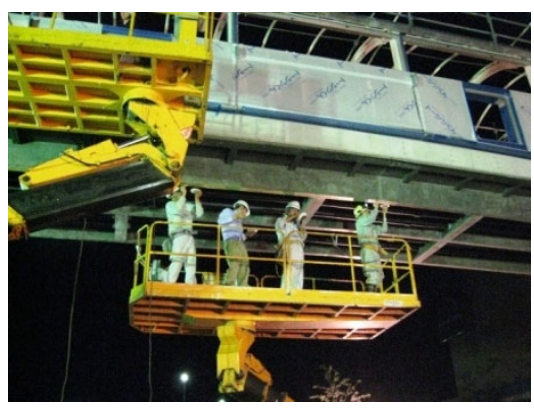

Fig. 13 Optical fiber sensor installation on girder.

Reinforcing the concrete with randomly distributed smooth fibers could successfully increase the durability and strength of the FRC. However, the requirements for manufacture of the FRC are more exacting than those of the normal concrete; a small deviation from the specified requirements for the FRC can result in major deficiencies of the quality and thus in poor test results [10]. Therefore, the FRC quality control is a very important issue, and the crack can be an index of its quality; but, visual inspection is currently the only direct way to detect cracks. Alternatively, strain gauges are used to measure the changes in the strain induced by cracks, but they have obvious pitfalls in terms of the pointed strain measurement. The FRC material is crackcritical, and hence, fully distributed strain measurements are required to ensure the flawless structure.

\subsection{Periodic strain measurement}

Using the BOCDA-based sensor, the longitudinal strain distribution along the girder was periodically measured, as shown in Fig. 14. Table 1 tabulates the lists of five measurements with the temperature on the surface of the girder. In the measurement setting used here, the spatial interval was approximately $40 \mathrm{~mm}$, and the spatial resolution was $48 \mathrm{~mm}$. 


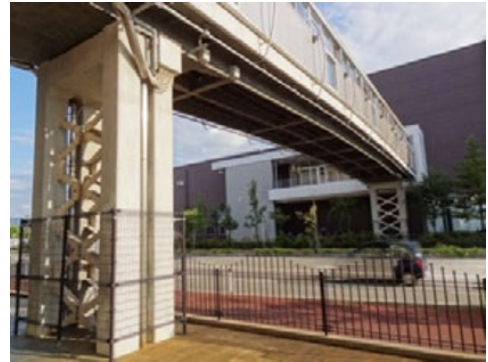

Fig. 14 Monitored pedestrian deck.

Table 1 Periodical strain measurement.

\begin{tabular}{ccc}
\hline Measurement & Date & Temperature $\left({ }^{\circ} \mathrm{C}\right)$ \\
\hline Initial & 28 Aug, 2007 & 25.0 \\
5 months & 29 Jan, 2008 & 2.5 \\
1 years & 4 Sep, 2008 & 25.6 \\
2 years & 17 Sep, 2009 & 20.2 \\
5 years & 13 Sep, 2013 & 25.7 \\
\hline
\end{tabular}

\subsection{Results and discussion}

The initial strain distribution in August 2007 was regarded as a baseline measurement, and the generated strain was calculated using the other four measurements, as shown in Figs. 15 (a) - 15 (d). These figures show $20-\mu \mathrm{m}$ crack-induced strain distribution models, which were re-calculated by taking the $48-\mathrm{mm}$ spatial resolution $(\Delta z=24 \mathrm{~mm})$, into (1). Although the whole range of the fiber shows scattering owing to the measurement precision error, $50 \times 10^{-6}-100 \times 10^{-6}$, the measurement region shows no consistent peak-shaped strain distribution like the $20-\mu \mathrm{m}$ crack model; therefore, one can at least conclude that the girder has no cracks, which are more than $20 \mu \mathrm{m}$ in width. Another important finding is that the surface mounted sensor can remain in situ and work for more than five years without any obvious deterioration (see Fig. 16).

It is well known that the optical fiber sensor is sensitive to the temperature, because the temperature change affects the refractive index of the optical fiber. Even if there is no strain on the fiber, the measurement is affected by temperature variations. In addition, the temperature has an impact on the thermal expansion of the member. In this study, it should be noted that the temperature-compensated strain distributions shown in Figs. 15 (a) - 15 (d) were based on the assumption that the both sides of measurement region around roller shoes must be zero strain because of the stress independence. Strain measurements shown in Fig. 15 (a), which were carried out in winter, apparently indicate the compressive strain all over the measurement region owing to the low temperature. The compressive strain might be attributed to the concrete creep and shrinkage with aging; in fact, the other three measurements indicate that the gradual compressive strain might occur mainly in $1 / 4$ and $3 / 4$ parts of the span. For more precise quantitative evaluation of gradual strain-changing events, the further temperature compensation method is needed.

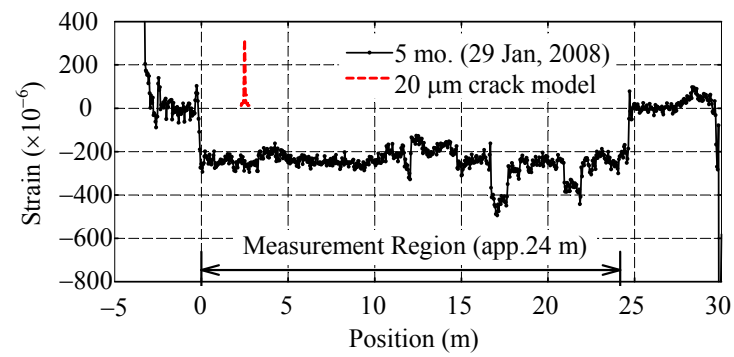

(a) 5 months

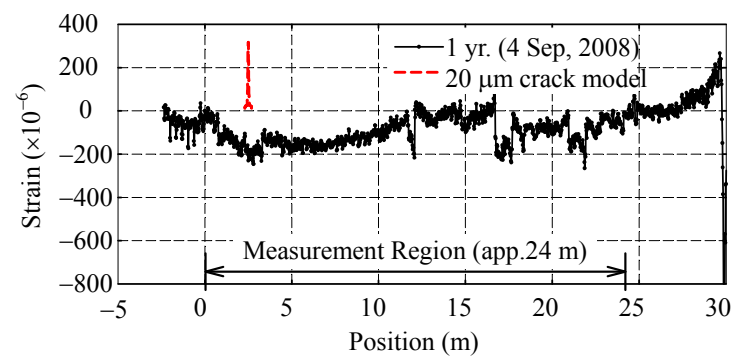

(b) 1 years

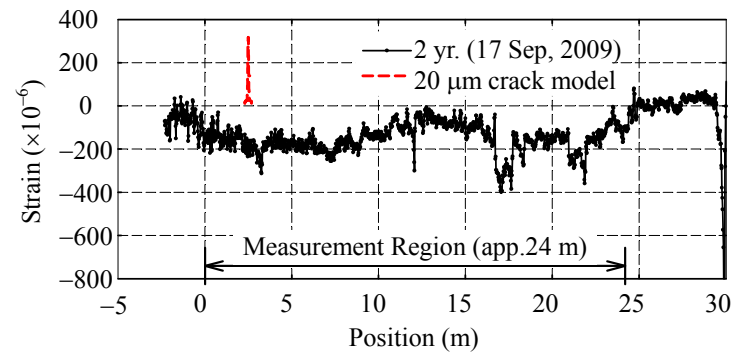

(c) 2 years

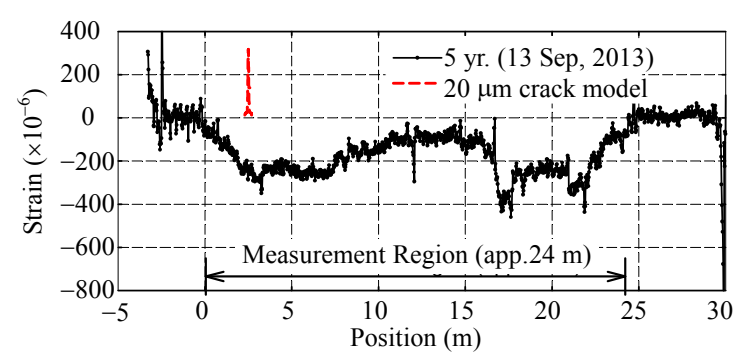

(d) 5 years

Fig. 15 Strain distribution measured by BOCDA. 


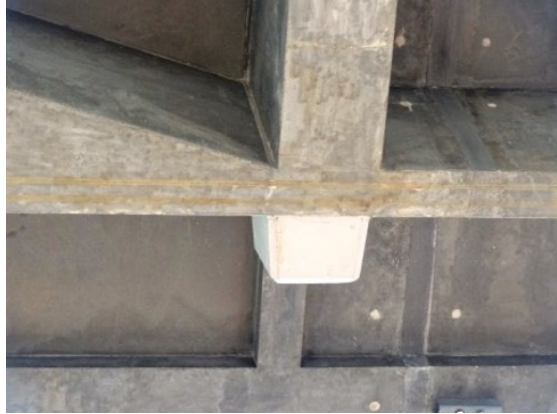

Fig. 16 Mounted five-year-old optical fiber.

\section{Summary and conclusions}

Focusing on crack identification, experimental studies were carried out using the BOCDA-based strain sensor. Firstly, considering the buffering effect of sensor's surrounding materials and the measurement sensitivity, a single-crack induced strain distribution model was proposed. Secondly, it was experimentally confirmed by the FRC beambending test that the width and location of discrete cracks could be determined by the proposed model. Finally, the sensor was applied to a pedestrian deck for five years, and they confirmed the absence of cracks. This study shows BOCDA's excellent potential for the local damage assessment. The technical obstacle to SHM still remains; however, the long-term monitoring would contribute to comprehend the behavior and cause of the crack in the future.

\section{Acknowledgment}

We gratefully acknowledge the continuous support provided by Prof. Hotate, the University of Tokyo. This study was supported partially by KAKENHI Grant-in-Aid for Young Scientists (A) (Grant No. 21681023).

Open Access This article is distributed under the terms of the Creative Commons Attribution License which permits any use, distribution, and reproduction in any medium, provided the original author(s) and source are credited.

\section{References}

[1] Y. Fujino and T. Takada, "Risk assessment, management and monitoring of infrastructure systems," in Stock management for sustainable urban regeneration, vol. 4. Y. Fujino and T. Noguchi, Ed. Japan: Springer Japan, 2009, pp. 233-253.

[2] B. Glisic and D. Inaudi, Fibre optic methods for structural health monitoring. UK: Wiley, 2007, pp. 19-40.

[3] K. Hotate and S. S. Ong, "Distributed fiber Brillouin strain sensing by correlation-based, continuous-wave technique, cm-order spatial resolution and dynamic strain measurement," in Proc. SPIE, vol. 4920, pp. 299-310, 2002.

[4] H. Naruse, Y. Uchiyama, T. Kurashima, and U. Shuji, "River levee change detection using distributed fiber optic strain sensor," IEICE Transactions on Electronics, vol. 83, no. 3, pp. 462-467, 2000.

[5] T. Vorster, K. Soga, R. Mair, P. Bennett, A. Klar, and C. Choy, "The use of fiber optic sensors to monitor pipeline response to tunneling," in GeoCongress 2006: Geotechnical Engineering in the Information Technology Age, Atlanta, USA, pp. 1-6, 2006.

[6] M. Imai, R. Nakano, T. Kono, T. Ichinomiya, S. Miura, and M. Mure, "Crack detection application for fiber reinforced concrete using BOCDA-based optical fiber strain sensor," Journal of Structural Engineering, vol. 136, no. 8, pp. 1001-1008, 2010.

[7] H. Niu and Z. S. Wu, "Peeling-off criterion for FRP-strengthened R/C flexural members," in Proc. of International Conference on FRP Composites in Civil Engineering, vol. 1, pp. 571-578, 2001.

[8] G. Duck and M. LeBlanc, "Arbitrary strain transfer from a host to an embedded fiber-optic sensor," Journal of Smart Materials and Structures, vol. 9, no. 4, pp. 492-497, 2000.

[9] M. Imai and M. Feng, "Sensing optical fiber installation study for crack identification using a stimulated Brillouin-based strain sensor," Structural Health Monitoring, vol. 11, no. 5, pp. 501-509, 2012.

[10] Z. Li and Y Zhang, "High-performance concrete," in Handbook of structural engineering, 2nd ed, W. F. Chen and E. M. Lui, Ed. USA: CRC Press, 2005, pp. 15-1-15-58. 